\title{
Theoretical study of ferroelectric triglycine sulphate (TGS) crystal in external electric fields
}

\author{
Trilok Chandra Upadhyay, Ashish Nautiyal ${ }^{*}$ \\ Department of Physics, School of Sciences, H.N.B. Garhwal University (A Central University), \\ Srinagar (Garhwal), Uttarakhand - 246174, India \\ *E-mail address: andehradun@yahoo.com
}

\begin{abstract}
A modified two sub-lattice pseudospin-lattice coupled mode model of Mitsui et al [Phys. Rev., 111 (1958) 1259] by adding third, fourth order phonon anharmonic interaction and external electric field terms has been applied to ferroelectric triglycine sulphate crystal. Electric field dependence of ferroelectric, dielectric and acoustical properties has been studied. With the help of double time temperature dependent Green's function method, expressions for shift, width, soft mode frequency, dielectric constant, loss tangent and acoustic attenuation have been derived. Numerically calculations have been made and results have been compared with experimental data reported by Bye et al [Ferroelectrics 4 (1974) 243] and Shreekumar et al [Ferroelectrics 160 (1994) 23] for TGS crystal and a good agreement has been observed.
\end{abstract}

Keywords: Ferroelectric; Green's function; Anharmonic; Dielectric constant; Loss tangent; Acoustic attenuation

PACS No.- 77.84.Fa

\section{INTRODUCTION}

Triglycine sulphate $\left(\mathrm{CH}_{2} \mathrm{NH}_{2} \mathrm{COOH}\right)_{3} \cdot \mathrm{H}_{2} \mathrm{SO}_{4}$ crystal has been widely studied since hundred decade before. Triglycine sulphate (TGS) crystal is one of the best pyroelectric materials and one of the rare ferroelectrics which shows isostructural phase transition. Triglycine sulphate crystal received universal attention ever since Mathias et al [1] have discovered its ferroelectric properties. It is uniaxial ferroelectrics with transition temperature $322 \mathrm{~K}$. Crystals are monoclinic in both polar and non-polar phases. Triglycine sulphate (TGS) crystallizes in the monoclinic system with space group P21 at room temperature. TGS crystals undergo a structural phase transition along the $\mathrm{b}$ axis, in which the space group is changed to $\mathrm{P} 21 / \mathrm{m}$. The thermal expansion is also an important thermodynamic property of crystals, since it is a result of the interactions and excitation of their constituent elements. The monoclinic $b$ axis is known to be parallel to spontaneous polarization direction. These $b$-cut plates of TGS crystals are used for room temperature infrared (IR) detectors, earth exploration, radiation monitoring and astronomical telescopes. TGS is reported to be the best choice among available materials as a sensitive element in pyroelectric sensors due to high pyroelectric coefficients, reasonably low dielectric constant and best figure of merit. The behavior of the domain structure of ferroelectrics in applied electric fields is most frequently studied by 
various indirect methods, because they provide information on the dynamics of the associated processes not only in near-surface layers but in the bulk of the sample as well.

The properties of ferroelectric crystals and their application as piezo-active and pyroactive elements depend strongly on their degree of unipolarity and conserving stability of this state. The lattice parameters of TGS crystal in ferroelectric phase are $a=9.42 \AA, b=12.64 \AA$, $\mathrm{c}=5.75 \AA$ and $\beta=110^{\circ} 23^{\prime}$ (at $\mathrm{T}<\mathrm{Tc}$ ) and in paraelectric phase (at $\mathrm{T}>\mathrm{Tc}$ ) remain unchanged. Large crystals can be easily grown from water solution. Experimental works on TGS have been initiated by Hoshino et al [2]. X-ray diffraction studies about crystal structure of TGS have been determined quite early. Since then many experimental studies on dielectric, ferroelectric, thermal and scattering properties of TGS crystal have been done. Recently Sun et al [3] have done growth and study about pyroelectric properties of pure and doped TGS crystal. Arago and Gonzalo [4] have done hysteresis curve studies of TGS crystal. Tolstekhina et al [5] have done atomic force microscopy domain structure on nano scale studies. Costache et al [6] have done pyroelectric properties of pure and alanine doped TGS crystals. Yamaguchi et al [7] have done dilatometric study on monoclinic crystal of TGS at quite low temperatures. Bye et al [8] have measured long ago, dielectric constant and spontaneous polarization of TGS crystal in presence of external electric field. Shreekumar et al [9] studied the acoustic dissipation in TGS crystal in external electric fields.

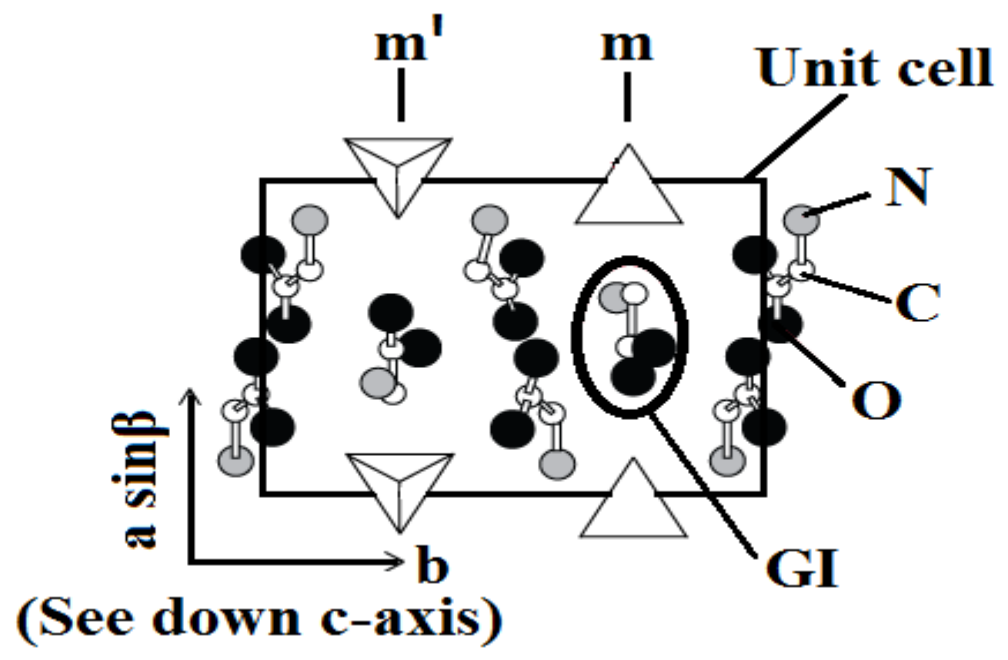

Fig. 1. Projection of the structure of TGS crystal along c-direction.

Theoretical studies of ferroelectric and dielectric properties of TGS crystal were initiated by Gonzalo et al [10] who used Ising type model. Tello and Hernandez et al [11] have used a tunneling pseudospin model. Blinc et al [12] have applied two-sublatiice pseudospin model [13] to TGS crytal. Chaudhuri et al [14] have used this model and added lattice coupling terms and phonon anharmonic interactions terms. These authors decoupled the correlations and disappeared from their expressions. Moreover, they have not studied effect of electric field on ferroelectric, dielectric and acoustical properties of TGS crystal. In their final calculations they have put $\mathrm{K}=0$ which is not correct.

In the present study we have modifed two-sublattice pseudospin lattice coupled mode model [14] by adding third and fourth order phonon anharmonic interaction terms [15] and external electric field terms. We have derived expressions for ferroelectric mode frequency 
dielectric constant, loss tangent and acoustic attenuation for TGS crystal with the help of double time temperature dependent Green's function method [16] and modified Hamiltonian. We have fitted model values in theoretical expression for soft mode frequency, dielectric constant, loss tangent and acoustic attenuation. Their temperature and electric field dependences are calculated. Theoretical results are compared with experimental results of Bye et al [8] and Shreekumar [9] et al.

\section{THEORY}

\section{1. Model Hamiltonian}

For ferroelectric TGS crystal, the two sublattice pseudospin lattice coupled mode model [14] is modified with third and fourth phonon anharmonic interaction terms [15] and electric field terms which is expressed as

$$
\begin{aligned}
& H=-2 \Omega \sum_{i}\left(S_{1 i}^{x}+S_{2 i}^{x}\right)-\sum_{i j} J_{i j}\left[\left(S_{1 i}^{z} S_{2 i}^{z}\right)+\left(S_{2 i}^{z} S_{2 i}^{z}\right)\right] \\
& -\sum_{i j} K_{i j}\left(S_{1 i}^{z} S_{2 i}^{z}\right)-2 \mu E \sum_{i}\left(S_{1 i}^{z}+S_{2 i}^{z}\right) \\
& -\sum_{i k} V_{i k}\left(S_{1 i}^{z} A_{k}\right)-\sum_{i k} V_{i k}\left(S_{2 i}^{z} A_{k}^{+}\right) \\
& +\frac{1}{4} \sum_{k} \omega_{k}\left(A_{k} A_{k}^{+}+B_{k} B_{k}^{+}\right) \\
& +\sum_{k_{1} V_{2}}^{(3)}\left(k_{1}, k_{2}, k_{3}\right) A_{k_{1}} A_{k_{2}} A_{k_{3}}+\sum_{k_{1} k_{2} k_{3} k_{4}}^{(4)}\left(k_{1}, k_{2}, k_{3}, k_{4}\right) A_{k_{1} A_{k} A_{k_{3}} A_{k}},
\end{aligned}
$$

Where, $\Omega$ is proton tunneling frequency, $\mathrm{S}_{1}{ }^{\mathrm{z}}$ and $\mathrm{S}_{1}{ }^{\mathrm{x}}$ are components of pseudospin variable, $\mathrm{J}_{\mathrm{ij}}$ is interaction between same lattice and $\mathrm{K}_{\mathrm{ij}}$ is interaction between different lattices. $\mu$ is dipole moment of $\mathrm{O}-\mathrm{H} \cdot \mathrm{O}$ bond, $\mathrm{E}$ is external electric field, $\mathrm{V}$ is spin lattice interaction and $A_{k}$ and $B_{k}$ are position and momentum operators, $\omega_{k}$ is the harmonic phonon frequency $V^{(3)}$ and $\mathrm{V}^{(4)}$ are third and fourth order atomic force constant.

\section{2. Green's function, shift and width}

Following Zubarev [15], we consider the evaluation of Green's function:

$$
\mathrm{G}_{\mathrm{ij}}\left(\mathrm{t}-\mathrm{t}^{\prime}\right)=\left\langle\left\langle\mathrm{S}_{1 \mathrm{i}}^{\mathrm{Z}}(\mathrm{t}) ; \mathrm{S}_{1 \mathrm{j}}^{\mathrm{Z}}\left(\mathrm{t}^{\prime}\right)\right\rangle\right\rangle=-\mathrm{i} \theta\left(\mathrm{t}-\mathrm{t}^{\prime}\right)\left\langle\left[\mathrm{S}_{1 \mathrm{i}}^{\mathrm{Z}}(\mathrm{t}) ; \mathrm{S}_{1 \mathrm{j}}^{\mathrm{Z}}\left(\mathrm{t}^{\prime}\right)\right]\right\rangle,
$$

where $\theta\left(\mathrm{t}-\mathrm{t}^{\prime}\right)$ is unit step function which is zero $\mathrm{t}<\mathrm{t}^{\prime}$ and unity for $\mathrm{t}>\mathrm{t}^{\prime}$. The angular bracket $<\ldots .>>$ denotes ensemble average over a grand canonical ensemble. Differentiating Green 
Function [Eq.(2)] with respect to time $t$ and $t$ ' respectively twice using Hamiltonian (1). Apply Fourier transforming and putting in the Dyson's equation form, one obtains:

$$
G_{i j}(\omega)=\frac{\Omega\left\langle S_{1 i}^{x}\right\rangle}{\pi\left(\omega^{2}-4 \widetilde{\Omega}^{2}-P(\omega)\right)}
$$

where

$$
\begin{aligned}
& \widetilde{\Omega}^{2}=a^{2}+b^{2}+b c \\
& a=2 J\left\langle S_{1}^{z}\right\rangle+K\left\langle S_{2}^{z}\right\rangle+2 \mu E \\
& b=2 \Omega \\
& c=2 J\left\langle S_{1}^{x}\right\rangle+K\left\langle S_{2}^{x}\right\rangle
\end{aligned}
$$

and

$$
P(\omega)=\frac{\pi^{2}}{\Omega^{2}\left\langle S_{1 i}{ }^{x}\right\rangle^{2}}\left\langle\left\langle F_{i}(t), F_{j}\left(t^{\prime}\right)\right\rangle\right\rangle
$$

In Eq.(8) contain higher order Green's Functions $\left\langle\left\langle F_{i}(t), F_{j}\left(t^{\prime}\right)\right\rangle\right\rangle$ which are decoupled by using scheme $<$ abcd $>=<\mathrm{ab}><\mathrm{cd}>+<\mathrm{ac}><\mathrm{bd}>+<\mathrm{ad}><\mathrm{bc}>$. The simpler Green's functions of $P(\omega)$ then evaluated in the zero ${ }^{\text {th }}$-order approximation. Substituting various such Green's functions $P(\omega)$ is evaluated. When $P(\omega)$ is resolved these are called shift and width respectively. In this way shift $\Delta(\omega)$ is obtained as

$$
\begin{aligned}
\Delta(\omega) & =\frac{a^{4}}{2 \Omega\left(\omega^{2}-\widetilde{\Omega}^{2}\right)}+\frac{b^{2} c^{2}}{4 \Omega \widetilde{\Omega}}+\frac{V_{i k}^{2} N_{k} a^{2}}{2 \Omega\left(\omega^{2}-\widetilde{\Omega}^{2}\right)}+\frac{4 \mu^{2} E^{2} a^{2}}{2 \Omega\left(\omega^{2}-\widetilde{\Omega}^{2}\right)} \\
& +\frac{2 V_{i k}^{2}\left\langle S_{1 i}^{x}\right\rangle \omega_{k} \delta_{k k^{i}}\left(\omega^{2}-\widetilde{\widetilde{\omega}}_{k}^{2}\right)}{\left[\left(\omega^{2}-\widetilde{\widetilde{\Omega}}_{k}^{2}\right)^{2}+4 \omega_{k}^{2} \Gamma_{k}^{2}(\omega)\right]}
\end{aligned}
$$

and width is $\Gamma(\omega)$

$$
\begin{aligned}
\Gamma(\omega)= & \frac{\pi a^{4}}{4 \Omega \widetilde{\Omega}}[\delta(\omega-\widetilde{\Omega})-\delta(\omega+\widetilde{\Omega})] \\
& +\frac{b^{2} c^{2}}{4 \Omega \widetilde{\Omega}}[\delta(\omega-\widetilde{\Omega})-(\omega+\widetilde{\Omega})]
\end{aligned}
$$




$$
\begin{aligned}
& +\frac{V_{i k}^{2} N_{k} a^{2}}{4 \Omega \widetilde{\Omega}}[\delta(\omega-\widetilde{\Omega})-\delta(\omega+\widetilde{\Omega})] \\
& +\frac{2 \pi \mu^{2} E^{2} a^{2}}{4 \Omega \widetilde{\Omega}}[\delta(\omega-\widetilde{\Omega})-\delta(\omega+\widetilde{\Omega})], \\
& +\frac{4 V_{i k}^{2}\left\langle S_{1 i}^{x}\right\rangle \omega_{k}\left(\omega^{2}-\widetilde{\widetilde{\omega}}_{k}^{2}\right)}{\left[\left(\omega^{2}-\widetilde{\widetilde{\omega}}_{k}^{2}\right)^{2}+4 \omega_{k}^{2} \Gamma_{k}^{2}(\omega)\right]}
\end{aligned}
$$

In the Eq.(10) and (12) $\widetilde{\widetilde{\omega}}_{k}$ is renormalized phonon frequency and $\Gamma_{k}(\omega)$ is phonon width in the Green's function $G_{k k^{\prime}}\left(t-t^{\prime}\right)=<<A_{k}(t) ; A_{k^{1}}\left(t^{\prime}\right)>>$ is evaluated using last three terms of Hamiltonian (1). The phonon Green's function is obtained as

$$
\begin{aligned}
& G_{k k^{\prime}}(\omega)=\frac{\omega_{k} \delta_{k k^{\prime}}}{\pi\left[\omega^{2}-\widetilde{\Omega}^{2}-2 \Omega i \Gamma(\omega)\right]} \\
& \widetilde{\widetilde{\omega}}_{\mathrm{k}}^{2}=\widetilde{\omega}_{\mathrm{k}}^{2}+2 \omega_{\mathrm{k}} \Delta_{\mathrm{k}}(\omega), \\
& \widetilde{\omega}_{k}^{2}=\widetilde{\omega}_{k}^{2}+A_{k}(T)
\end{aligned}
$$

phonon shift $\Delta_{k}(\omega)$ and width $\Gamma_{k}(\omega)$ are obtained as

$$
\begin{aligned}
\Delta_{k}(\omega) & =\operatorname{Re} a l P_{k}(\omega) \\
& =18 P \sum_{k_{1} 2}\left|V^{(3)}\left(k_{1}, k_{2},-k\right)\right|^{2} \\
& \frac{\omega_{\mathrm{k} 1} \omega_{\mathrm{k} 2}}{\widetilde{\omega}_{\mathrm{k} 1} \widetilde{\omega}_{\mathrm{k} 2}}\left\{\left(\mathrm{n}_{\mathrm{k} 1}+\mathrm{n}_{\mathrm{k} 2}\right) \frac{\widetilde{\omega}_{\mathrm{k} 1}+\widetilde{\omega}_{\mathrm{k} 2}}{\omega^{2}-\left(\widetilde{\omega}_{\mathrm{k} 1}+\widetilde{\omega}_{\mathrm{k} 2}\right)^{2}}\right. \\
& \left.+\left(\mathrm{n}_{\mathrm{k}_{2}}-\mathrm{n}_{\mathrm{k}_{1}}\right) \frac{\widetilde{\omega}_{\mathrm{k} 1}+\widetilde{\omega}_{\mathrm{k} 2}}{\omega^{2}-\left(\widetilde{\omega}_{\mathrm{k} 1}+\widetilde{\omega}_{\mathrm{k} 2}\right)^{2}}\right\} \\
& +48 P \sum_{k 1_{1} k_{2 k}}\left|V^{(4)}\left(k_{1} k_{2}, k_{3,}-k\right)\right|^{2} \frac{\omega_{k_{1}} \omega_{k 2} \omega_{k 3}}{\widetilde{\omega}_{k_{1}} \widetilde{\omega}_{k_{2}} \widetilde{\omega}_{k 3}}
\end{aligned}
$$




$$
\begin{aligned}
& \left\{\left(1+n_{k_{1}} n_{k_{2}}+n_{k_{2}} n_{k_{3}}+n_{k_{3}} n_{k_{1}}\right) \frac{\widetilde{\omega}_{k_{1}}+\widetilde{\omega}_{k 2}+\widetilde{\omega}_{k 3}}{\omega^{2}-\left(\widetilde{\omega}_{k_{1}}+\widetilde{\omega}_{k_{2}}+\widetilde{\omega}_{k_{3}}\right)^{2}}\right. \\
& +3\left(1-\mathrm{n}_{\mathrm{k}_{2}} \mathrm{n}_{\mathrm{k}_{1}}+\mathrm{n}_{\mathrm{k} 2} \mathrm{n}_{\mathrm{k}_{3}}-\mathrm{n}_{\mathrm{k}_{3}} \mathrm{n}_{\mathrm{k}_{1}}\right) \\
& \left.\frac{\widetilde{\omega}_{\mathrm{k}_{1}}+\widetilde{\omega}_{\mathrm{k} 2}+\widetilde{\omega}_{\mathrm{k} 3}}{\omega^{2}-\left(\widetilde{\omega}_{\mathrm{k}_{1}}+\widetilde{\omega}_{\mathrm{k}_{2}}+\widetilde{\omega}_{\mathrm{k} 3}\right)^{2}}\right\}
\end{aligned}
$$

and phonon width

$$
\begin{aligned}
& \Gamma_{k}(\omega)=\operatorname{Im} P_{k}(\omega) \\
& =9 \pi \sum_{k_{1} k_{2}}\left|V^{(3)}\left(k_{1}, k_{2},-k\right)\right|^{2} \frac{\omega_{k_{1}} \omega_{k_{2}}}{\widetilde{\omega}_{k_{1}} \widetilde{\omega}_{k_{2}}} \\
& \left\{\left(n_{k_{1}}+n_{k_{2}}\right)\left[\delta\left(\omega+\widetilde{\omega}_{k_{1}}+\widetilde{\omega}_{k_{1}}\right)\right]\right. \\
& -\delta\left(\omega-\widetilde{\omega}_{\mathrm{k}_{1}}-\widetilde{\omega}_{\mathrm{k}_{1}}\right)+\text { (higher terms) } \\
& \left.+\left(\mathrm{n}_{\mathrm{k}_{2}}-\mathrm{n}_{\mathrm{k}_{1}}\right)\left\lfloor\delta\left(\omega+\widetilde{\omega}_{\mathrm{k}_{1}}+\widetilde{\omega}_{\mathrm{k}_{2}}\right)-\delta\left(\omega+\widetilde{\omega}_{\mathrm{k}_{1}}+\widetilde{\omega}_{\mathrm{k}_{2}}\right)\right\rfloor\right\} \\
& +48 \pi \sum_{\mathrm{k}_{1} \mathrm{k}_{2} \mathrm{k}_{3}}\left|\mathrm{~V}^{(4)}\left(\mathrm{k}_{1}, \mathrm{k}_{2}, \mathrm{k}_{3},-\mathrm{k}_{4}\right)\right|^{2} \frac{\omega_{\mathrm{k}_{1}} \omega_{\mathrm{k}_{2}} \omega_{\mathrm{k}_{3}}}{\tilde{\omega}_{\mathrm{k}_{1}} \tilde{\omega}_{\mathrm{k}_{2}} \tilde{\omega}_{\mathrm{k}_{3}}} \\
& \mathrm{X}\left\{\left(1+\mathrm{n}_{\mathrm{k}_{1}} \mathrm{n}_{\mathrm{k}_{2}}+\mathrm{n}_{\mathrm{k}_{2}} \mathrm{n}_{\mathrm{k}_{3}}+\mathrm{n}_{\mathrm{k}_{3}} \mathrm{n}_{\mathrm{k}_{4}}\right)\right. \\
& \left.X \mid \delta\left(\omega+\widetilde{\omega}_{k_{1}}+\widetilde{\omega}_{k_{2}}+\widetilde{\omega}_{k_{3}}\right)-\delta\left(\omega-\widetilde{\omega}_{k_{1}}-\widetilde{\omega}_{k_{2}}-\widetilde{\omega}_{k_{3}}\right)\right] \\
& +3\left(\mathrm{n}_{\mathrm{k}_{1}} \mathrm{n}_{\mathrm{k}_{2}}+\mathrm{n}_{\mathrm{k}_{2}} \mathrm{n}_{\mathrm{k}_{3}}-\mathrm{n}_{\mathrm{k}_{3}} \mathrm{n}_{\mathrm{k}_{4}}\right)+3\left(\mathrm{n}_{\mathrm{k}_{1}} \mathrm{n}_{\mathrm{k}_{2}}+\mathrm{n}_{\mathrm{k}_{2}} \mathrm{n}_{\mathrm{k}_{3}}-\mathrm{n}_{\mathrm{k}_{3}} \mathrm{n}_{\mathrm{k}_{4}}\right) \\
& \left.X\left[\delta\left(\omega+\widetilde{\omega}_{k_{1}}-\widetilde{\omega}_{k_{2}}-\widetilde{\omega}_{k_{3}}\right)-\delta\left(\omega-\widetilde{\omega}_{k_{1}}+\widetilde{\omega}_{k_{2}}+\widetilde{\omega}_{k_{3}}\right)\right]\right\}
\end{aligned}
$$

The Green's function (3) after putting values of $P(\omega)$ becomes

$$
G_{i j}(\omega)=\frac{\Omega\left\langle S_{1 i}^{x}\right\rangle \delta_{i j}}{\pi\left(\omega^{2}-\hat{\Omega}^{2}-2 \Omega i \Gamma(\omega)\right)}
$$

where

$$
\hat{\Omega}^{2}=\widetilde{\Omega}^{2}+2 \Omega \Delta(\omega)
$$


In Eq. (17) $\widetilde{\Omega}$ is given by Eq. (4) and $\Delta_{k}(\omega)$ is given by Eq. (14). When Eq. (17) is solved self consistently by putting value of $\Delta_{k}(\omega)$ one gets

$$
\hat{\Omega}_{ \pm}^{2}=\frac{1}{2}\left(\widetilde{\widetilde{\omega}}_{\mathrm{k}}^{2}+\widetilde{\widetilde{\Omega}}^{2}\right) \pm \frac{1}{2}\left[\left(\widetilde{\widetilde{\omega}}_{\mathrm{k}}^{2}-\widetilde{\widetilde{\Omega}}^{2}\right)^{2}+8 \mathrm{~V}_{\mathrm{ik}}^{2}\left\langle\mathrm{~S}_{1 \mathrm{i}}^{\mathrm{x}}\right\rangle \Omega\right]^{1 / 2}
$$

In Eq. (18) $\hat{\Omega}$ is soft mode frequency which becomes zero at transition temperature causing phase transition in TGS crystal.

We may obtain expression for transition temperature by putting $\Delta_{k}(\omega) \rightarrow 0$ as $T \rightarrow T_{C}$ in Eq. (18) above:

$$
\mathrm{T}_{\mathrm{c}}=\frac{\eta}{2 \mathrm{k}_{\mathrm{B}} \tanh ^{-1}\left(\frac{\eta^{3}}{4 \Omega^{2} \mathrm{~J}^{\prime}}\right)}
$$

where

$$
\eta^{2}=(2 J-K)^{2} \sigma^{2}+4 \Omega^{2}+(2 \mu E)^{2}
$$

and

$$
\left.J *=(2 J+K)+\frac{2 V_{i k}^{2} \widetilde{\widetilde{\omega}}_{k}^{2}}{\left[\widetilde{\widetilde{\omega}}_{k}^{4}+4 \omega_{k} \Gamma_{k}^{2}\right.}\right]
$$

In Eq. (21) ${J_{i j}}^{*}$ is renormalized exchange interaction constant.

\section{3. Dielectric constant and loss tangent}

Following Zubarev et al [15], the electric susceptibility $\chi$ is related to Green's function $G_{i j}(\omega+i x)$ as

$$
\chi(\omega)=-\lim _{X \rightarrow 0} 2 \pi N \mu^{2} G_{i j}(\omega+i x)
$$

The dielectric constant $\varepsilon$ is related to $\chi(\omega)$ as:

$$
\in=1+4 \pi \chi
$$

with the help of Eq. (22), (23) and (16) dielectric constant ( $\varepsilon$ ) is obtained as $\varepsilon>>>1$, 


$$
\in(\omega)=\left(-8 \pi N \mu^{2}\right) \frac{\left\langle S_{1}^{x}\right\rangle \Omega}{\left[\left(\omega^{2}-\hat{\Omega}^{2}\right)^{2}+4 \Omega^{2} \Gamma^{2}\right]}
$$

The dissipation of power in dielectric when it is exposed to A.C. electric field is expressed as loss tangent:

$$
\tan \delta=\frac{\epsilon^{\prime \prime}}{\epsilon^{\prime}}
$$

or

$$
\tan \delta=-\frac{2 \Omega \Gamma(\omega)}{\left(\omega^{2}-\hat{\Omega}^{2}\right)}
$$

Acoustic attenuation in TGS crystal will be

$$
\alpha=\frac{\Gamma}{v}
$$

where $\Gamma$ is lattice frequency width and $\mathrm{v}$ is the velocity of sound in the crystal.

\section{NUMERICAL CALCULATION AND RESULTS} [13],

The model values of various quantities for TGS crystal are given by Chaudhuri et al

$$
\begin{aligned}
& T_{C}=49^{0} C, \Omega=0.1\left(\mathrm{~cm}^{-1}\right), J=340\left(\mathrm{~cm}^{-1}\right), K=170\left(\mathrm{~cm}^{-1}\right), V_{i k}=10\left(\mathrm{~cm}^{-3 / 2}\right), \\
& \omega_{k}{ }^{2}=0.59\left(\mathrm{~cm}^{2}\right), N_{\mu}=2.22 \times 10^{18} \mathrm{esu}, A_{k}=10.2, C=3007 \mathrm{~K}
\end{aligned}
$$

Table 1. Values of $2 \mu E$ for TGS crystal

\begin{tabular}{|c|c|c|c|c|c|c|c|c|c|c|}
\hline $\mathbf{E}(\mathbf{k V} / \mathbf{c m}$ & 1 & 2 & 3 & 4 & 5 & 6 & 7 & 8 & 9 & 10 \\
\hline $2 \mu E\left(\mathrm{~kJ} / \mathrm{cm}^{3}\right)$ & 0.0003756 & 0.0007512 & 0.0011268 & 0.0015024 & 0.001878 & 0.0022536 & 0.0026292 & 0.0030048 & 0.0033804 & 0.003756 \\
\hline
\end{tabular}

Table 2. Ultrasonic velocity in TGS crystal at room temperature. (Yu-Fen et al [17])

\begin{tabular}{|c|}
\hline Pure TGS crystal $\left(\mathbf{1 0}^{\mathbf{3}} \mathbf{~} \mathbf{~} / \mathbf{s}\right)$ \\
\hline $\mathrm{Vx}=5.218$ \\
\hline $\mathrm{Vy}=4.371$ \\
\hline $\mathrm{Vz}=4.068$ \\
\hline
\end{tabular}


The temperature and field dependence of ferroelectric mode frequency, dielectric constant, loss tangent and acoustic attenuation have been calculated for TGS crystal using model values and Table 1 and 2. Calculated values are shown in Figs. 2-5.

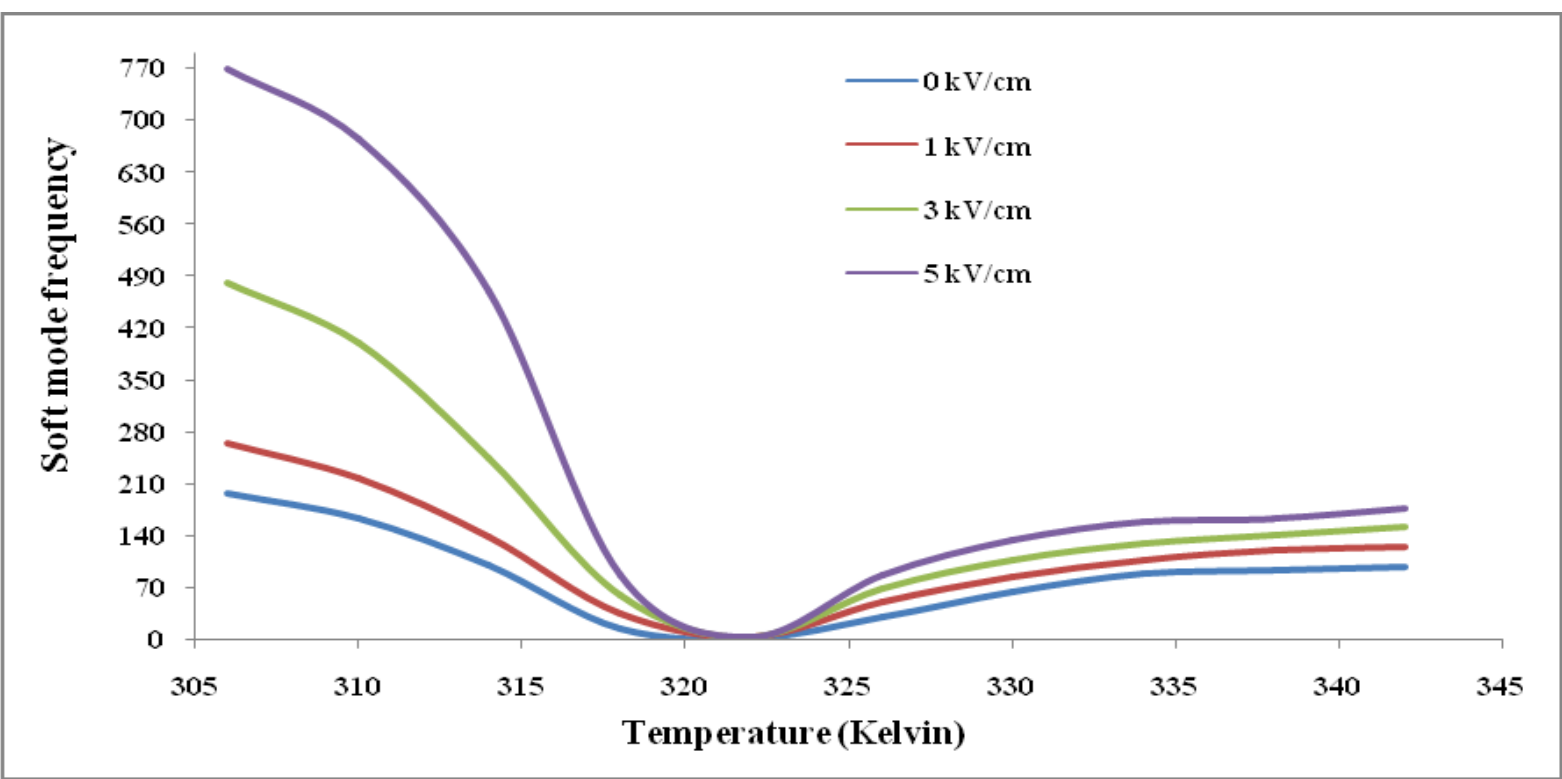

Fig. 2. Calculated temperature dependence of ferroelectric mode frequency of TGS crystal in presence of electric field.

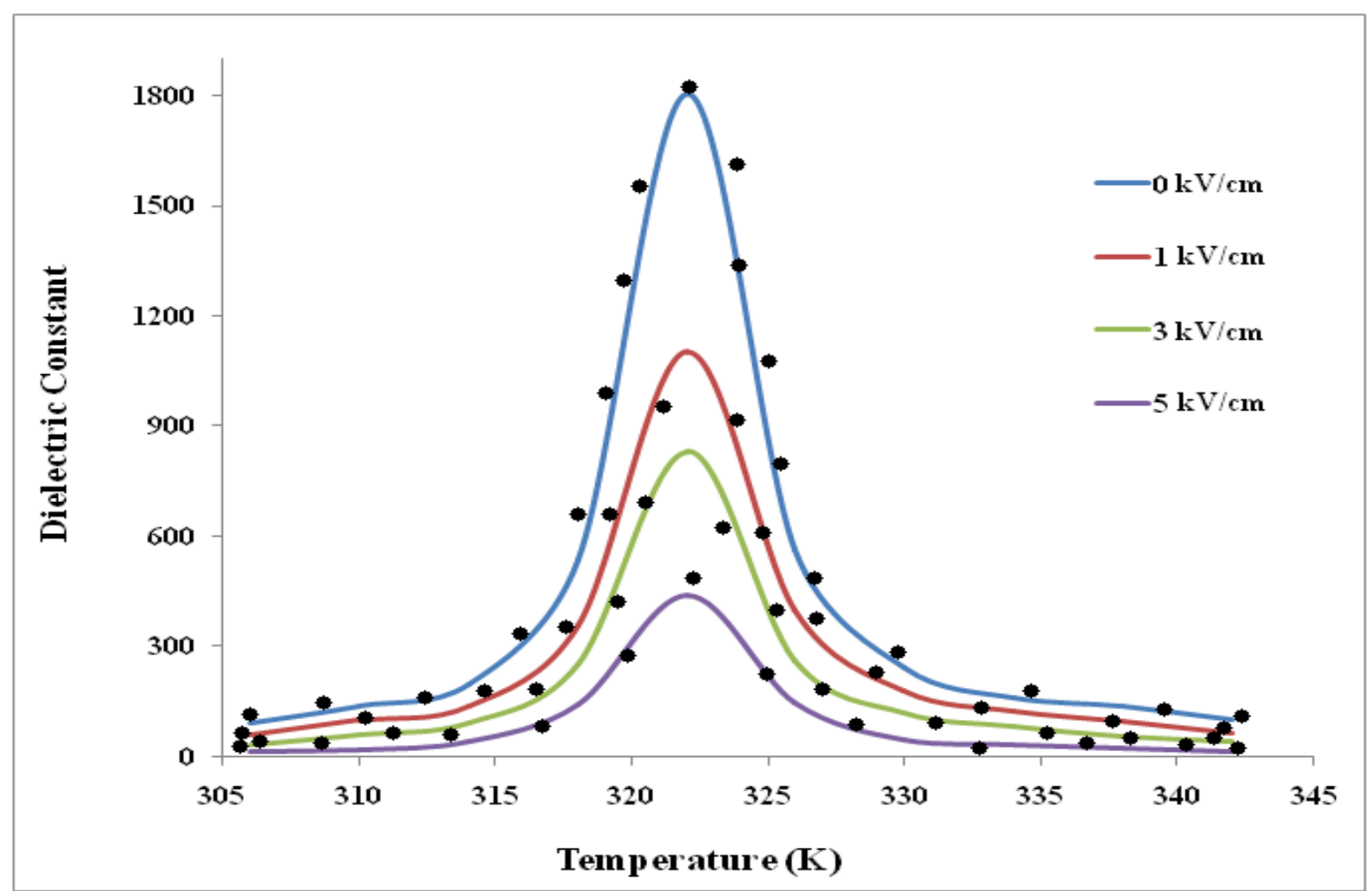

Fig. 3. Calculated temperature dependence of dielectric constant of TGS crystal in presence of electric field (- our calculation; - experimental data of Bye et al [8]). 


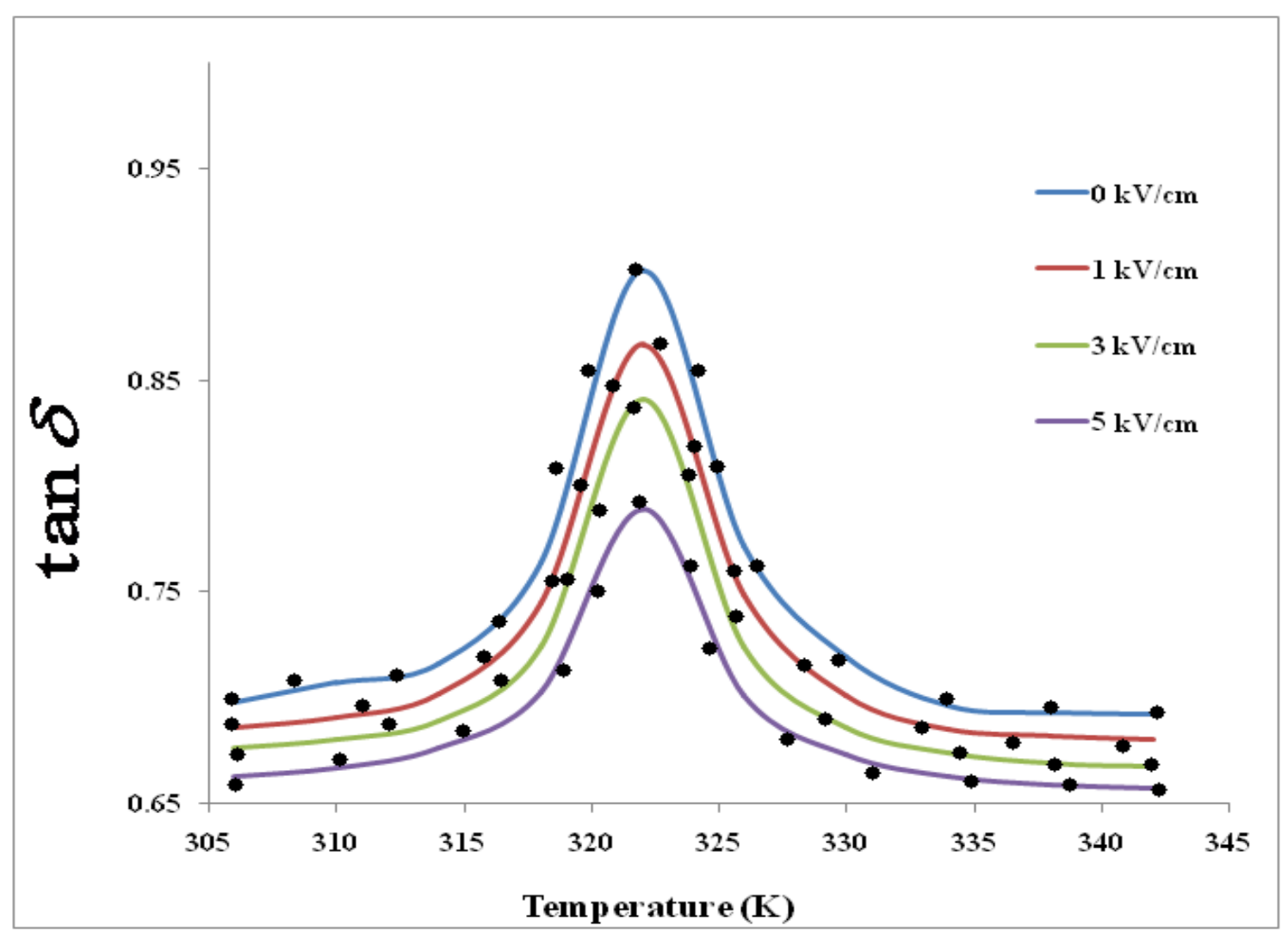

Fig. 4. Calculated temperature dependence of loss $\operatorname{tangent}(\tan \delta$ ) of TGS crystal in presence of electric field ( - our calculation; • experimental data of Bye et al [8]).

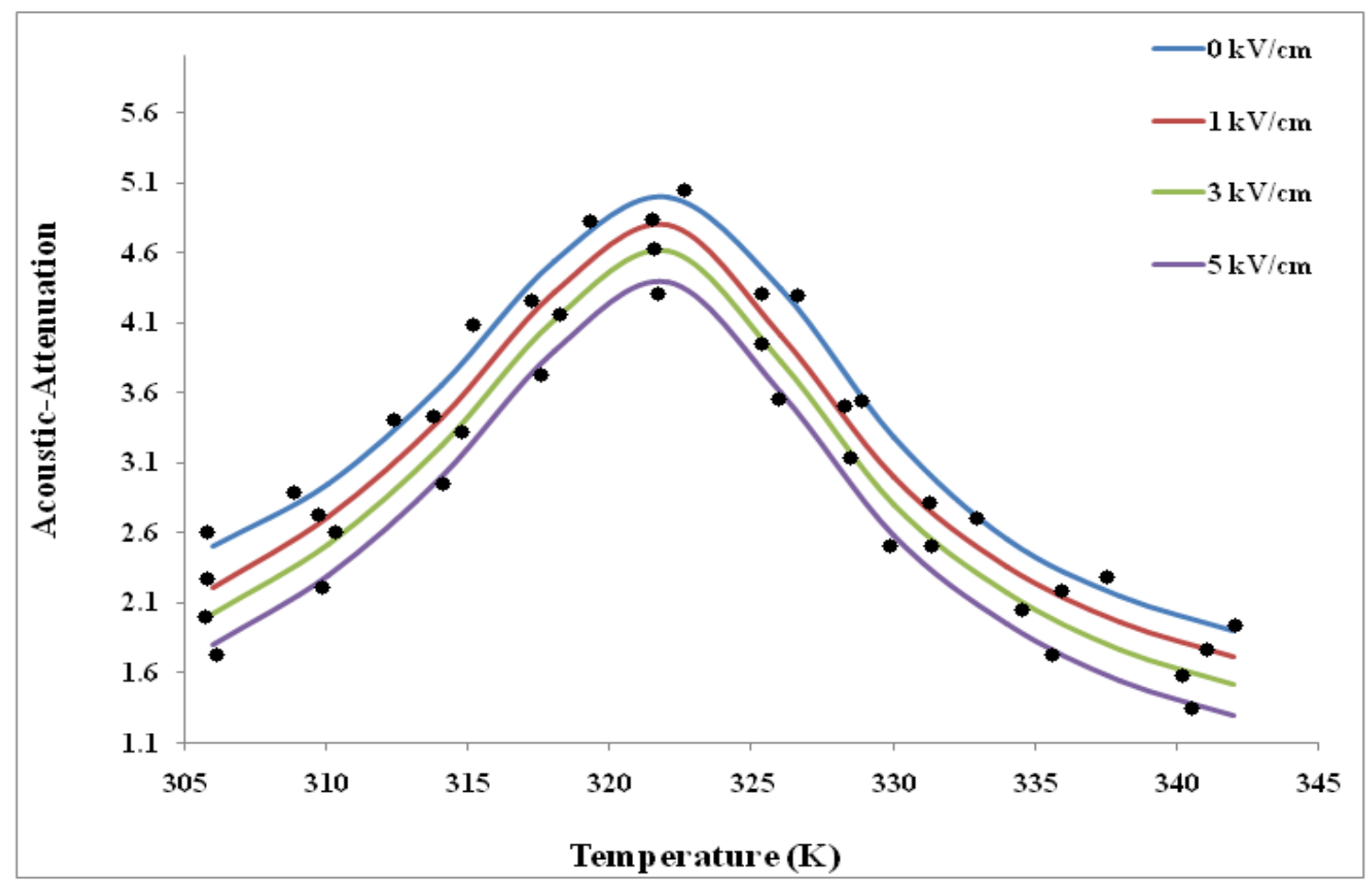

Fig. 5. Calculated temperature dependence of acoustic attenuation of TGS crystal in presence of electric field (- our calculation; - experimental data of Shreekumar et al [9]). 
Our theoretical results agree with experimentally reported results Bye et al [8] and Shreekumar et al [9] who has studied the effect of electric field on dielectric constant and acoustic attenuation in TGS crystal. It can be seen from our theoretical data that the ferroelectric mode frequency increases with increasing electric field strength [see Eq. (18) and (4)].

The dielectric constant, loss tangent and acoustic attenuation decrease with increase in electric field strength [see Eq. (24) and (26)]. The observations are in good agreement with experimental finding of Bye et al [8] and Shreekumar et al [9] for TGS crystal.

\section{DISCUSSION}

In the present work, by considering two sublattice pseudospin-lattice coupled mode model modified by adding third and fourth order phonon anharmonic interaction terms and external electric field terms expressions have been derived for ferroelectric mode frequency, dielectric constant, loss tangent and acoustic attenuation for TGS crystal. The double time temperature dependent Green's function has been used for the derivation of response function. Earlier authors have not considered electric field terms.

They have decoupled the corrections terms at early stages and finally disappeared from their expressions. Our expression for $\widetilde{\Omega}$ is similar to their expression except electric field term $\left(\mu^{2} E^{2}\right)$.

However our ferroelectric mode frequency $\hat{\Omega}$ contains extra terms $\Delta(\omega)$ [given by Eq. (14)], as compared to ferroelectric mode frequency derived by Chauduri et al [13]. These authors have not considered electric field terms. Our loss tangent contains extra terms contained in $\Gamma(\omega)$. Our calculated results at phase transition temperature $\left(\mathrm{T}_{\mathrm{c}}\right)$ are more realistic i.e. soft mode frequency $\left(10^{-1}\right.$ order $)$, dielectric permittivity $\left(10^{3}\right.$ order $)$, loss tangent $\left(10^{-1}\right.$ order) and acoustic attenuation $\left(10^{0}\right.$ order) while in external electric fields results show that ferroelectric mode frequency increases, dielectric permittivity, loss tangent and acoustic attenuation decreases with increasing strength of external electric field. In the presence of constant bias electric field crystal behaves as damped crystal and it has changed force constants between ions, due to which vibrational frequency of ions/atoms increases or time period of oscillations of ions decreases.

The electric field dues the exclusion of domains in crystal. As a result this decreases dielectric constant, loss tangent and corresponding acoustic attenuation the crystal.

\section{CONCLUSION}

We have applied two-sublattice pseudospin lattice coupled mode model modified with third- and fourth order- phonon anharmonic interaction terms and external electric field terms explains the ferroelectric, dielectric and acoustical properties of TGS crystal.

Present expressions can also explain ferroelectric and dielectric properties of similar crystals such as Rochelle salt, lead hydrogen phosphate and potassium dihydrogen phosphate crystals in presence of electric field. 


\section{ACKNOWLEDGMENT}

We are grateful to Prof. R. P. Gairola (Univ. Head of Physics Department, H. N. B. G. U., Srinagar Garhwal), Prof. B. S. Semwal (H. N. B. G. U., Srinagar Garhwal) for his kind blessings and valuable suggestions and to Prof. U. C. Naithani (HOD, H. N. B. G. U., Pauri Garhwal), Prof. Vinay Gupta (Delhi Univ.), Prof. K. K. Verma (R. M. L. A. U., Faizabad) for encouragement. we are thankful to Mr. Sandeep Sharma for discussion and to library staff Mr. Pawan Bisht and Mr. Jhaldiyal for help in library, H. N. B. G. U., Srinagar Garhwal and chauras campuses.

\section{References}

[1] B. T. Mathias, C. E. Miller, Remeika, Phys. Rev. 104 (1956) 449.

[2] S. Hoshino, Y. Okaya, R. Pepinsky, Phys. Rev. 115 (1959) 323.

[3] X. Sun, M. Wang, Q. P. Pan, W. Shi, C. S. Fang, Crys Res. Technol. 34 (1994) 1251.

[4] C. Arago, J. A. Ganzalo, J. Phys. C 12 (2000) 3737.

[5] A. L. Tolstikhina, N. V. Belugina, S. A. Shikin, Ultramicroscopy 82 (2000) 149.

[6] M. Costache, I. Matel, L. Pintilic, H. V. Alexandra, C. Berbeearu, Opto \& Adv. Mat. 3 (2001) 75 .

[7] T. Yamaguchi, Nakatani, T. Kikuta, T. Kurihama, T. Mitsui, Y. Seimiya, S. Yoshizawa, F. Shionizu, M. Takashige, Ferroelectrics 337 (2006) 3125.

[8] K. L. Bye, P. W. Whipps, E. T. Keve, Ferroelectrics 4 (1972) 253.

[9] R. Shreekumar, J. Philip, Ferroelectrics 160 (1994) 23.

[10] J. A. Gonzalo, Phys. Rev. B1 (1970) 3125.

[11] M. J. Tello, E. Hernandez, J. Phys. Soc. Jpn. 35 (1973) 1289.

[12] R. Blinc, S. Detoni, M. Pintar, Phys. Rev. 124 (1961) 1036.

[13] T. Mitsui, Phys. Rev. 111 (1958) 1259.

[14] B. K. Chaudhuri, K. R. Chaudhari, S. Benerjie, Phys. Rev. B 38 (1988) 689.

[15] B. S. Semwal, P. K. Sharma, Progr. Theor. Phys. 51 (1974) 639.

[16] D. N. Zubarev, Sov. Phys. Usp. 3 (1960) 320.

[17] Yu-Fen Chang, Utrasonic study on effects of ultraviolet irradiation on $\alpha$-alanine doped triglycine sulphate, department of physics, National Cheng Kung University, Ph.D. thesis work (2003), p 46. 